\title{
MMP-2/9-oriented combinations enhance antitumor efficacy of EGFR/HER2-targeting fusion proteins and gemcitabine
}

\author{
YE QIN, XIU-JUN LIU, LIANG LI, XU-JIE LIU, YI LI, RUI-JUAN GAO, \\ RONG-GUANG SHAO and YONG-SU ZHEN \\ Institute of Medicinal Biotechnology, Chinese Academy of Medical Sciences and \\ Peking Union Medical College, Beijing 100050, P.R. China
}

Received February 17, 2014; Accepted April 2, 2014

DOI: $10.3892 /$ or.2014.3169

\begin{abstract}
To increase the antitumor efficacy, in the present study, we proposed several settings of matrix metalloproteinase (MMP)-2/9-oriented combinations that comprise the MMP-2/9-targeting fusion protein $\mathrm{dFV}$-LDP and the MMP inhibitor doxycycline (DOX) in association with EGFR/ HER2-bispecific fusion protein Ec-LDP-Hr, its enediyneenergized analogue Ec-LDP-Hr-AE, and gemcitabine (GEM). The expressions of various fusion proteins were detected by western blot analysis. Proliferation and migration inhibition of cells were determined by MTT and Transwell assay, respectively. The binding capability of dFv-LDP and Ec-LDP-Hr to cancer cells was examined by ELISA, cell immunofluorescence coimmunoprecipitation and confocal assays. Animal experiments were set to investigate the antitumor efficacy of various combinations against colorectal carcinoma HCT-15 xenograft in athymic mice. These two targeting proteins dFv-LDP and Ec-LDP-Hr had strong binding capabilities and antiproliferation effects on various cancer cell lines. Enhanced therapeutic efficacy in vivo was observed in the MMP-2/9-targeting fusion protein $\mathrm{dFv}-\mathrm{LDP}$ integrated combinations including: i) $\mathrm{dFv}-\mathrm{LDP}$ and Ec-LDP-Hr, ii) $\mathrm{dFv}-\mathrm{LDP}$ and enediyneenergized fusion protein Ec-LDP-Hr-AE, iii) dFv-LDP and Ec-LDP-Hr-AE plus DOX, and iv) dFv-LDP and GEM plus DOX against colorectal cancer HCT-15 xenograft in athymic mice. In setting iii, DOX (20 mg/kg), dFv-LDP $(20 \mathrm{mg} / \mathrm{kg})$ and Ec-LDP-Hr-AE $(0.3 \mathrm{mg} / \mathrm{kg})$ alone suppressed tumor growth by $35,49.7$ and $67.5 \%$, respectively. The combination of $\mathrm{dFV}$-LDP and Ec-LDP-Hr-AE was $75.1 \%$. Furthermore, this combination plus DOX showed stronger efficacy with an inhibitory rate of $82.7 \%$. In setting iv, the combination of dFv-LDP and GEM suppressed tumor growth by $66.3 \%$. Notably, the
\end{abstract}

Correspondence to: Professor Yong-Su Zhen, Institute of Medicinal Biotechnology, Chinese Academy of Medical Sciences and Peking Union Medical College, 1 Tiantan Xili, Beijing 100050, P.R. China E-mail: zhenys@public.bta.net.cn

Key words: MMP-2/9, EGFR/HER2, gemcitabine, doxycycline, targeting combinations, colorectal cancer tumor inhibitory rate of the $\mathrm{dFv}-\mathrm{LDP} / \mathrm{GEM} / \mathrm{DOX}$ combination reached $85.5 \%$, producing initial shrinkage after the first administration. The MMP-2/9-oriented combination strategy that employs the MMP-2/9-targeting antibody-based fusion protein and the small molecular inhibitor DOX as the basic composed agents may enhance antitumor efficacy in association with the EGFR/HER2-targeting fusion protein and GEM. This multiple targeting approach may be useful for enhancing antitumor efficacy against colorectal cancer.

\section{Introduction}

Conventional anticancer drugs often lack specificity, resulting in toxicities to healthy tissues and a poor therapeutic index (1). Increasingly more research has recently focused on antibody-based drugs including monoclonal antibodies (mAbs), antibody-drug conjugates (ADCs), immunotoxins and immunoliposomes (1). These antibody-based targeting drugs not only inhibit tumor cell proliferation by interfering with ligand binding or by blocking receptor dimerization (2), but they also maintain the ability to collaborate with chemotherapeutic drugs (3). Evidence has shown that combinations comprising antibody-based targeting therapies to several targets [such as epithelial growth factor receptor (EGFR), HER2] overexpressed in cancer cells, were more effective than the single agent (4-6). Integrated combinations comprising antibodybased drugs, chemotherapy and other agents are promising for cancer therapy.

As therapeutic targets of cancer, EGFR and HER2, the subgroup I and II of the ErbB family of receptors closely related receptor tyrosine kinases, are known to play an essential role in regulating cell proliferation and differentiation. The overexpression of EGFR and HER2 observed in many human tumors and their synergistic interaction in the transformation of cells make these receptors important targets for the development of new targeted therapeutics $(7,8)$. Dual inhibition of EGFR and HER2 was proposed for cancer based on their observed overexpression in cancer cells (9-11). In fact, various anticancer drugs that target EGFR have been designed and are used in the adjuvant chemotherapeutic treatment of metastatic disease (6).

Moreover, it has been reported that matrix metalloproteinase (MMP)-2 and -9 (also called gelatinases) are highly 
expressed in several cancer areas compared to normal areas (12-14). The expression rate of MMP-2 in the colorectal tumor border is much higher than in the tumor center (15), indicating that MMP-2 in tumor borders has key roles in tumor invasion. The increased levels of MMP-9 in cancer have also been attributed to cancer invasion and metastasis (12). MMP-2/9 overexpression is associated with poorer overall and progression-free survival in patients with colorectal cancer $(13,14)$. Some biomedical evidence has shown that MMP-2/9 could be promising therapeutic targeting markers.

We have reported that Ec-LDP-Hr-AE, a ligand-based (EGFC-loop fragment targeting EGFR) and antibody-based $\left(\mathrm{V}_{\mathrm{H}} \mathrm{CDR} 3\right.$ region of anti-HER2 antibody C6.5) bispecific fusion protein energized with enediyne, showed highly potent cytotoxicity to a variety of cancer cells in vitro and was highly effective against carcinoma xenograft in vivo (16). In addition, a gelatinase-targeting tandem $\mathrm{dFv}$-based fusion protein $\mathrm{dFv}$-LDP was generated in this laboratory (17) (Fig. 1A). Our previous study showed that these two types of antibody-based fusion proteins had strong binding abilities to cancer cells and their enediyne-energized analogues had potent antitumor effects against cancer xenografts that overexpress EGFR/ HER2 or MMP-2/9, respectively.

In addition to targeting therapy, chemotherapy is also needed to improve the outcome of advanced cancer patients. Recently, several studies suggested that gemcitabine (GEM) had a modest activity in cancer patients and may be an option in patients $(18,19)$. Also, the MMP inhibitor doxycycline (DOX) could also be an adjuvant therapeutic agent in several types of cancer (20-22). In the present study, we hypothesized that an MMP-2/9-oriented drug combination strategy may achieve enhancement of antitumor efficacy. The present study was performed with several settings of combinations based on the MMP-2/9-targeting fusion protein $\mathrm{dFv}$-LDP plus the MMP inhibitor drug DOX in vivo, and include: i) dFv-LDP plus the EGFR/HER2-bispecific fusion protein Ec-LDP-Hr, ii) dFv-LDP plus the EGFR/HER2-bispecific and enediyneenergized fusion protein Ec-LDP-Hr-AE, iii) dFv-LDP plus Ec-LDP-Hr-AE and DOX, and iv) dFv-LDP plus GEM and DOX. The results indicated that the MMP-2/9-targeting fusion protein $\mathrm{dFv}-\mathrm{LDP}$ acts as a versatile agent to increase antitumor efficacy against colorectal cancer xenograft when combined with EGFR/HER2-targeted fusion protein or the chemotherapeutic GEM. Further enhance efficacy can be achieved by the addition of the MMP inhibitor DOX.

\section{Materials and methods}

Cell culture and materials. The human colorectal cancer HCT-15 and fibrosarcoma HT1080 cells were cultured in RPMI-1640 (HyClone, Logan, UT, USA). The media was supplemented with $10 \%$ fetal bovine serum (FBS; HyClone), $100 \mathrm{U} / \mathrm{ml}$ penicillin and $100 \mu \mathrm{g} / \mathrm{ml}$ streptomycin. Cells were routinely grown as monolayer in culture flasks and maintained in exponential growth to $\sim 90 \%$ confluence for experiments. GEM was purchased from Lilly France S.A. (Lille, France). DOX was purchased from Shanghai Yuanye Biotechnology Co., Ltd. The primary antibodies were purchased from Cell Signaling Technology, Inc. (Danvers, MA, USA). The second antibodies were purchased from Zhongshan Golden
Bridge Biotechnology (Beijing, China). Cell lysis buffer was purchased from GenStar Biosolutions Co. (Beijing, China).

Western blotting and coimmunoprecipitation analysis. Cells were lysed for $30 \mathrm{~min}$ in RIPA lysis buffer (GenStar Biosolutions Co). Extracts were clarified by centrifugation at $10,000 \mathrm{rpm}$ for $20 \mathrm{~min}$ at $4^{\circ} \mathrm{C}$. Thirty micrograms of each total protein were applied on a 10\% SDS-PAGE and electroblotted onto polyvinylidene difluoride membranes (Millipore, Billerica, MA, USA). The membranes were blocked with $5 \%$ milk or $5 \%$ bovine serum albumin (BSA) before incubating with primary antibodies (diluted 1:1,000; Cell Signaling Technology) and then incubated with goat anti-mouse/rabbit peroxidase-coupled antibody (diluted 1:4,000). The membranes were washed with Tris-buffered saline with $0.5 \%$ Tween-20 between every step for five times. The specific bands were visualized with the Immobilon Western Chemiluminescent HRP Substrate kit (Millipore).

For coimmunoprecipitation assay, cells were lysed in cell lysis buffer for $30 \mathrm{~min}$ at $4^{\circ} \mathrm{C}$. One milligram of total protein from cell lysates was incubated with $100 \mu \mathrm{g}$ Ec-LDP-Hr or $\mathrm{dFv}$-LDP protein at $4^{\circ} \mathrm{C}$ and the mixtures were then incubated overnight with $1 \mu \mathrm{g}$ anti-EGFR, anti-HER2 or anti-MMP2 antibody at $4^{\circ} \mathrm{C}$; complexes were collected with protein $\mathrm{A}+\mathrm{G}$ agarose (Beyotime, Beijing, China) and the precipitates were washed five times with ice-cold phosphate-buffered saline (PBS). Then, proteins were released by boiling in sample buffer, followed by western blot analysis as described above. The fusion protein was detected by incubation of the membranes with anti-His-tag monoclonal antibody.

MTT assay. 3-(4,5-Dimethylthiazol-2-yl)-2,5-diphenyltetrazolium bromide (MTT) assay was used to determine cell proliferation. Cells were seeded in 96-well plates at a density of 3,000-5,000 cells/well, incubated in $37^{\circ} \mathrm{C}$ for $24 \mathrm{~h}$ and then exposed to different agents for 48 or $72 \mathrm{~h}$. MTT (Sigma) solution $(5 \mathrm{mg} / \mathrm{ml}, 20 \mu \mathrm{l})$ was added to each well and incubated for another $4 \mathrm{~h}$ at $37^{\circ} \mathrm{C}$. The supernatant was removed and $150 \mu \mathrm{l}$ DMSO were added to each well. The absorbance at $570 \mathrm{~nm}$ was measured by a microplate reader (Thermo Fisher Scientific). Growth inhibition was calculated as a percentage of the non-treated controls.

ELISA examination. Enzyme-linked immunosorbent assay (ELISA) was used for measuring the binding efficiency of tested proteins to colorectal cancer cells. Cells were seeded in 96-well plates at a density of 20,000 cells/well and incubated at $37^{\circ} \mathrm{C}$ for $24 \mathrm{~h}$ and were then fixed with methanol and blocked with $1 \%$ BSA for $1 \mathrm{~h}$ at room temperature. After washing with PBS, cells were supplemented with $100 \mu \mathrm{l}$ of 2 -fold dilutions of tested proteins ranging from 50 to $0.1 \mu \mathrm{mol} / 1$ and incubated at $37^{\circ} \mathrm{C}$ for $2 \mathrm{~h}$. A 2,500-fold dilution of His-Tag (2A8) mouse mAb (M20001L; Abmart, Omaha, NE, USA) and a 2,500-fold diluted horseradish peroxidase (HRP)-conjugated goat anti-mouse IgG (Zhongshan Golden Bridge Biotechnology) were used as the primary and the secondary antibodies, respectively. A total of $100 \mu 1$ of TMB (PA107-01, Tiangen, Beijing, China) was added as substrate solution, followed by terminating reaction with $2 \mathrm{~mol} / \mathrm{l} \mathrm{H}_{2} \mathrm{SO}_{4}$. The absorbance at $450 \mathrm{~nm}$ was measured using a microplate reader (Thermo 
A
dFv-LDP

\begin{tabular}{|l|l|l|}
\hline Fv & Fv & LDP \\
\hline
\end{tabular}
MMP-2/9 targeting

Ec-LDP-Hr

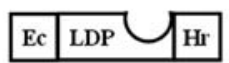

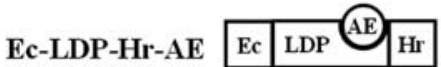

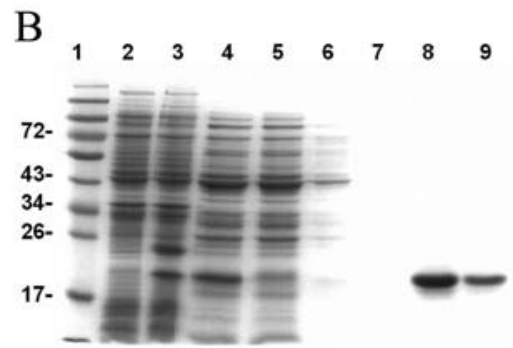

EGFR/HER2 targeting

Ec-LDP-Hr energized with enediyne

Figure 1. Preparation of fusion proteins dFv-LDP and Ec-LDP-Hr. (A) Schematic depictions of the tandem scFv-based fusion protein (dFv-LDP) and bispecific fusion protein Ec-LDP-Hr and its energized fusion protein (Ec-LDP-Hr-AE) in the present study. (B) SDS-PAGE analysis of the expression of Ec-LDP-Hr protein and purification of the protein by $\mathrm{Ni}^{+}$affinity chromatography. 1 , Low molecular weight protein markers $(\mathrm{kDa}) ; 2$, total cell protein fraction before IPTG induction; 3 , total cell protein fraction after IPTG induction; 4, protein extracted from $E$. coli periplasmic space; 5 , proteins unbound with Ni2 ${ }^{+}$column; 6 and 7, proteins washed with washing buffer; 8 and 9, Ec-LDP-Hr protein eluted with elution buffer. (C) SDS-PAGE analysis of the expression of dFv-LDP protein and purification of the protein by $\mathrm{Ni}^{+}$affinity chromatography. 1 , Low molecular weight protein marker (kDa); 2 , total cell protein fraction before IPTG induction; 3 and 4, total cell protein fraction after IPTG induction; 5, the soluble portion of recombinant Rosetta(DE3)pLysS pellet after sonication; 6, the soluble portion of recombinant Rosetta(DE3)pLysS pellet after sonication washed by 2 M urea; 7, the soluble portion of recombinant Rosetta(DE3)pLysS pellet after sonication washed by $6 \mathrm{M}$ urea; 8 , proteins unbound with Ni2 ${ }^{+}$column; 9 , proteins washed with washing buffer; 10 , dFv-LDP protein eluted with elution buffer.

Fisher Scientific, Bremen, Germany), and the affinity constant was calculated. All assays were carried out in triplicate.

ELISA was also used to detect the MMP-2 in cell culture media. The concentration of MMP-2 secreted from cells was determined by the ELISA kit according to the manufacturer's protocol (EK0459; Boster, Wuhan, China).

Cell immunofluorescence and confocal assays. Cell immunofluorescence was used to measure the binding efficiency of proteins to colorectal cancer cells. Seeded at 20,000 cells/well in 24-well plates, the cells were cultured for $24 \mathrm{~h}$. After washing with PBS, the cells were fixed with methanol and blocked with $1 \%$ BSA for $1 \mathrm{~h}$ at room temperature and were then incubated with $50 \mu \mathrm{mol} / 1$ proteins at $4^{\circ} \mathrm{C}$ overnight. Then, the cells were incubated with His-Tag (2A8) mouse $\mathrm{mAb}(1: 200 ; \mathrm{M} 20001 \mathrm{~L})$ at $37^{\circ} \mathrm{C}$ for $2 \mathrm{~h}$. After washing with PBS, cells were incubated with tetramethylrhodamine isothiocyanate (TRITC)-conjugated goat anti-mouse IgG (1:200; Zhongshan Golden Bridge Biotechnology) at room temperature for $1 \mathrm{~h}$ (avoiding light). The cells were then incubated with $300 \mathrm{nmol} / 1$ 4,6-diamidino-2-phenylindole (DAPI) to stain nuclei for $20 \mathrm{~min}$ at room temperature (avoiding light). After washing with PBS, each sample was analyzed by a fluorescence microscope and recorded by a camera.

The binding affinity of fusion protein Ec-LDP-Hr to HER2 on the HCT-15 cell surface was also analyzed by laser scanning confocal assay. Following the above-mentioned protocol to the step of incubation with TRITC-conjugated goat anti-mouse $\mathrm{IgG}$, cells were then washed with PBS and incubated with rabbit anti-HER $2 \mathrm{mAb}$ (\#2165; CST, Inc.) at $4^{\circ} \mathrm{C}$ overnight (avoiding light). Then, cells were washed with PBS and incubated with the Alexa Fluor 488-conjugated AffiniPure goat anti-rabbit IgG (ZF-0511; Zhongshan Golden Bridge Biotechnology) at room temperature for $2 \mathrm{~h}$ (avoiding light). Then, the cells were stained with DAPI at room temperature for 20 min (avoiding light). After washing with PBS, the images were observed under a laser scanning confocal microscope (Leica TCS SP2, Germany).

Transwell migration assay. The migration potential of HCT-15 cells was examined using Transwell inserts fitted with polycarbonate filter (8- $\mu \mathrm{m}$ available pore size, \#3422; Corning Inc., Corning, NY, USA). Cells in FBS-free medium $\left(5 \times 10^{5}\right.$ cells $\left./ \mathrm{ml}\right)$ were seeded in the upper compartment while the lower wells contained 10 or $20 \%$ FBS medium as a chemoattractant. Following 24 or $48 \mathrm{~h}$ incubation with $\mathrm{dFv}$-LDP and DOX, the cells in the upper chamber were removed with a cotton swab while other cells, which had passed through the filter on the underside of the membrane, were fixed with methanol, stained with $0.01 \%$ crystal violet and then captured at x40 magnification using the camera on invert microscope. Subsequently, the crystal violet was then dissolved with $33 \%$ acetic acid and the absorbance was measured at $450 \mathrm{~nm}$ by using a microplate reader.

Animal experiments. Antitumor experiments were carried out using the HCT-15 xenograft model of human colorectal carcinoma. Female athymic mice (BALB/c, nu/nu) were purchased from the Institute for Experimental Animals, Chinese Academy of Medical Science and Peking Union Medical College. The experiments were performed according to the regulation of Good Laboratory Practice for non-clinical 
A

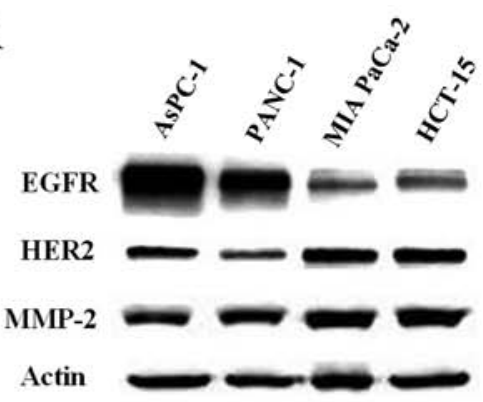

$\mathrm{C}$

HCT-15

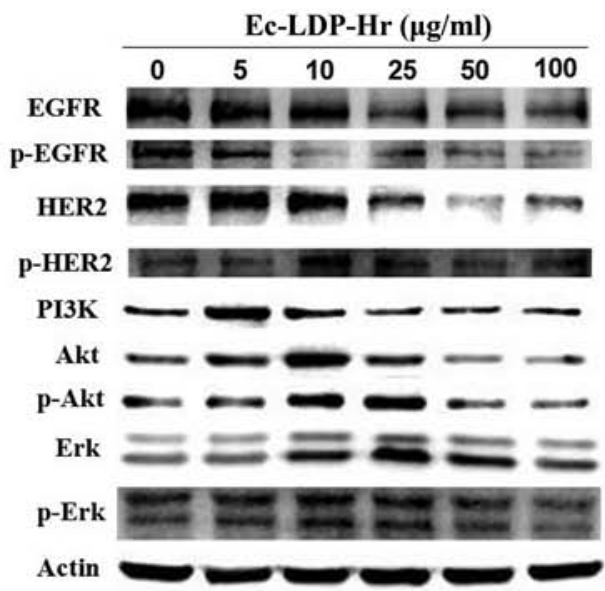

E

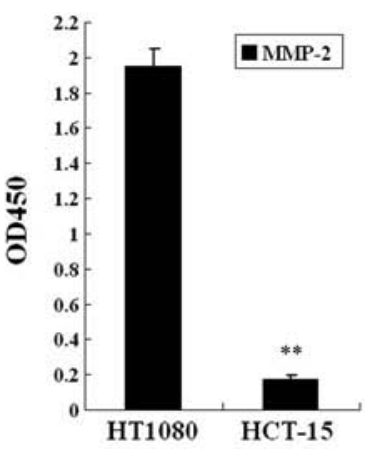

B

HCT-15

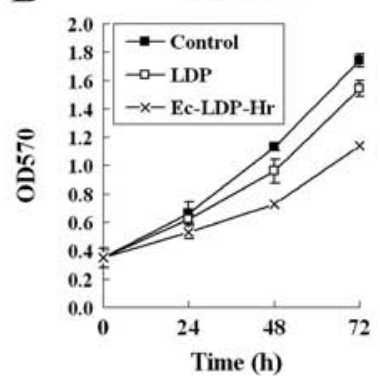

PANC-1

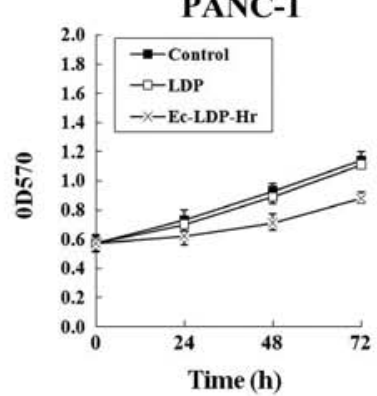

PANC-1

$\mathrm{D}$

$\operatorname{Ec}-\mathrm{LDP}-\mathrm{Hr}(\mu \mathrm{g} / \mathrm{ml})$

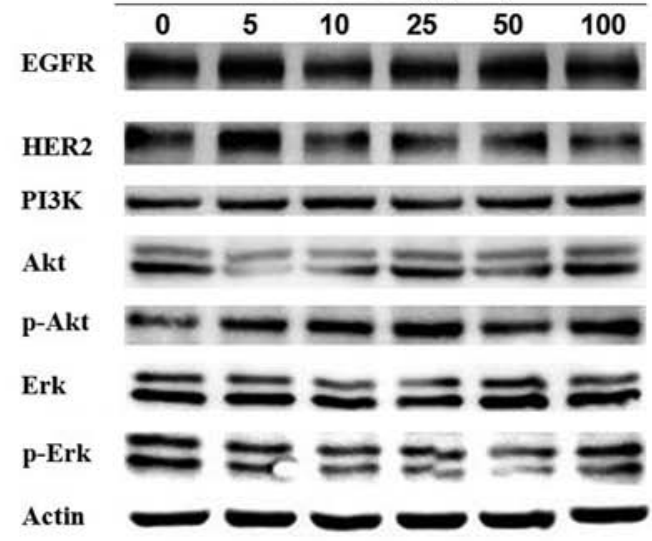

$\mathrm{F}$

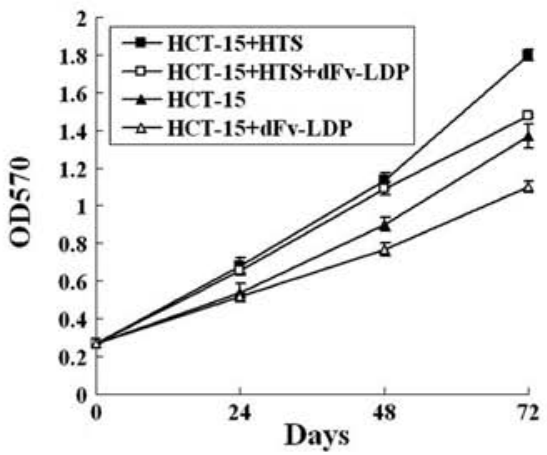

Figure 2. Fusion proteins dFv-LDP and Hc-LDP-Hr inhibit the proliferation of cancer cells. (A) Western blot analysis of the expression levels of EGFR, HER2 and MMP-2 in different human cancer cell lines. (B) Proliferation inhibition of HCT-15 and PANC-1 cancer cells by Ec-LDP-Hr (50 $\mu \mathrm{g} / \mathrm{ml})$ and LDP $(50 \mu \mathrm{g} / \mathrm{ml})$ at 24, 48 and $72 \mathrm{~h}$ determined by MTT assay. (C and D) Analysis the expression and phosphorylation levels of EGFR/HER2 signal pathway of HCT-15 and PANC-1 cancer cells treated with Ec-LDP-Hr by western blotting. (E) MMP-2 is highly secreted from fibrosarcoma HT1080 cells measured by ELISA. ${ }^{* *} \mathrm{P}<0.01$. (F) Proliferation promotion of HCT-15 cells conditioned with HT1080 culture surpernatant (HTS) was attenuated by dFv-LDP.

laboratory studies of drugs issued by the National Scientific and the Technologic Committee of People's Republic of China.

Animal experiments were performed with athymic mice weighing $18-20 \mathrm{~g}$. Mice were inoculated subcutaneously in the right flank with $1 \times 10^{7}$ HCT-15 cells suspended in $200 \mu 1$ PBS. Tumors grown after 3-4 weeks in donor animals were aseptically dissected and mechanically minced. Pieces of tumor tissue ( $2 \mathrm{~mm}$ in diameter) were transplanted subcutaneously in the right flank of mice by a trocar needle. When the tumors were established and reached $\sim 50-100 \mathrm{~mm}^{3}$ in size, the mice were randomly divided into groups $(n=6)$. The experimental mice were injected twice through the tail vein at an interval of 7 days. At the same time, one group of mice was given physiological saline as control. Every 2 days, tumor size was measured with a caliper, and tumor volume was calculated using the following formula: Tumor volume $\mathrm{V}=\left(\mathrm{axb}^{2}\right) / 2$, where $\mathrm{a}$ and $\mathrm{b}$ are the long and the perpendicular short diameters of the tumor, respectively. At the end of the experiment, all mice were weighted and sacrificed, and their tumors were excised. Tumors were weighed, and the tumor growth inhibition was calculated as follows: Tumor growth inhibition $(\%)=(1-\mathrm{T} / \mathrm{C}) \times 100 \%$. $\mathrm{T}$ is the mean tumor weight of treated group, whereas $\mathrm{C}$ represents the mean tumor weight of the control group. 
A

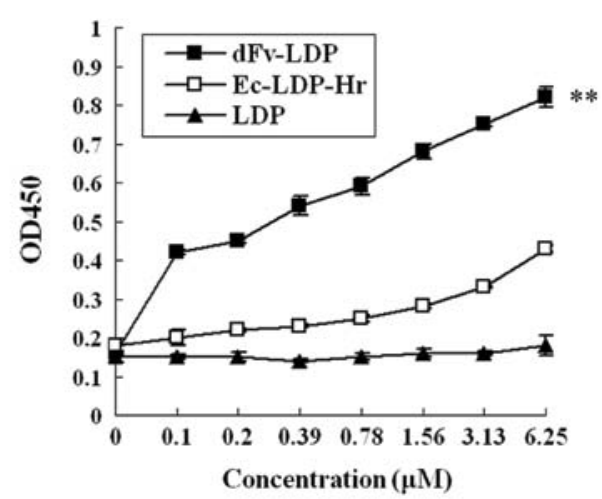

C

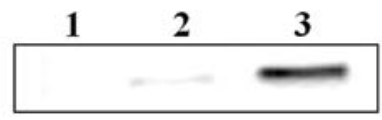

D

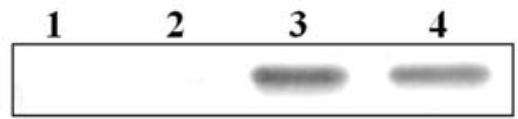

B
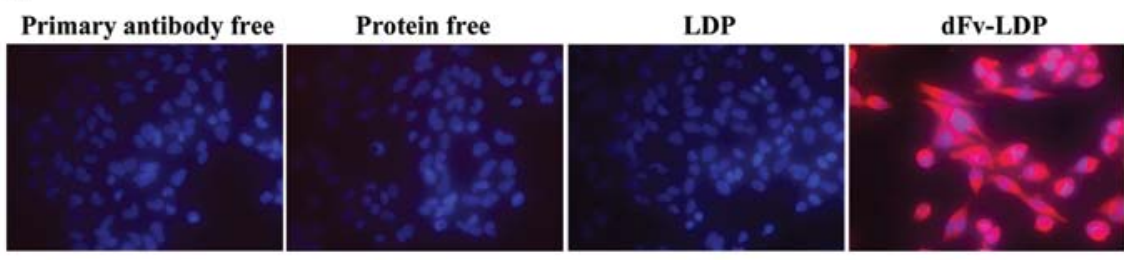

Ec-LDP-Hr

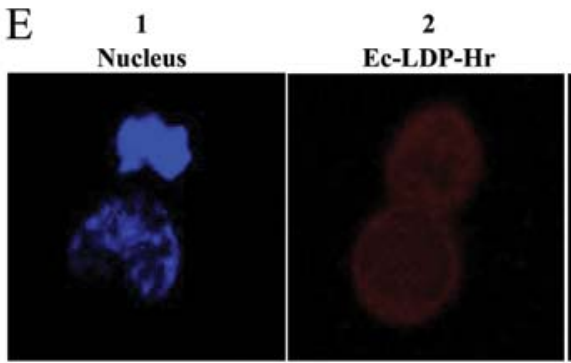
Anti-HER2 mAb
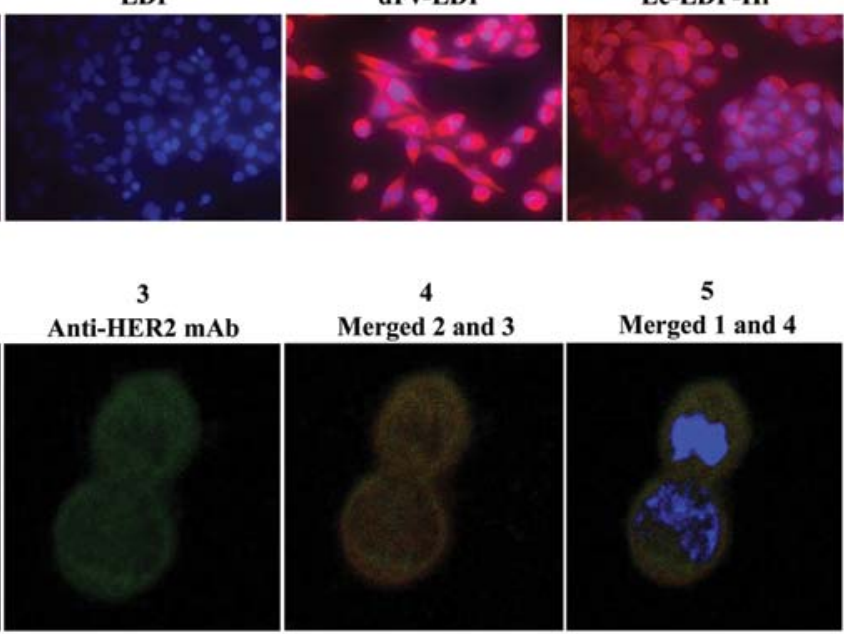

5

Merged 1 and 4

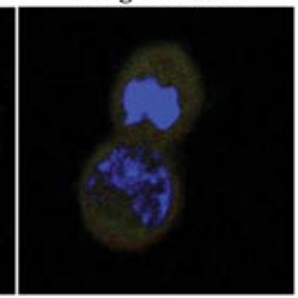

Figure 3. Fusion proteins dFv-LDP and Ec-LDP-Hr have strong binding ability to human colorectal cancer HCT-15 cells. (A) The binding affinity of dFv-LDP and Ec-LDP-Hr proteins with HCT-15 cells analyzed by ELISA. ${ }^{* *} \mathrm{P}<0.01$ compared with Ec-LDP-Hr and LDP. (B) Binding affinity of dFV-LDP and LDP with the HCT-15 cells analyzed by immunofluorescent cytochemical staining at $\mathrm{x} 400$. The red color represents dFv-LDP and Ec-LDP-Hr by mouse anti-His-Tag antibody and TRITC labeled anti-mouse IgG. Blue color indicates the nuclei stained with DAPI. (C and D) The binding specificity of dF-LDP and Ec-LDP-Hr proteins to MMP-2, EGFR and HER2 were verified by coimmunoprecipitation assay. Total proteins extracted from HCT-15 cells were incubated with PBS (lanes C1 and D1) or Ec-LDP-Hr protein (lanes C2-3 and D2-4). Subsequently, the mixture was incubated with either preimmune serum (lane C2 and D2), anti-MMP-2 (lane C3), anti-EGFR (lane D3), anti-HER2 (lane D4). The complexes formed were collected with protein A + G agarose and were analyzed by SDS-PAGE and immunoblotting with anti-His-tag monoclonal antibody. (E) Binding affinity of Ec-LDP-Hr with HER2 on HCT-15 cells analyzed by confocal microscopy. 1, Blue color indicates the nuclei stained with DAPI; 2, the red color represents Ec-LDP-Hr by mouse anti-His-Tag antibody and TRITC labeled anti-mouse IgG; 3, the green color represents HER 2 by mouse anti-HER2 antibody and FITC labeled anti-mouse IgG; 4, merged 2 and 3, yellow area represents the co-localization of HER2 and Ec-LDP-Hr; 5, merged 1 and 4.

Statistical analysis. Data are presented as the means \pm SD. Statistical comparisons between groups were executed by Student's t-test, and a significant difference was considered if $\mathrm{P}<0.05$. The correlation of binding capability among different tested proteins was analyzed using Pearson's $\chi^{2}$ test. P-value $<0.05$ was considered to indicate a statistically significant difference. Analysis was performed using the SPSS statistical software package for windows (version 17.0, SPSS, Inc. Chicago, IL, USA).

\section{Results}

Proliferation inhibition of cancer cells by Hc-LDP-Hr and $d F v-L D P$. The schematic depictions (Fig. 1A) represent the molecular constitution of the proteins and enediyne-energized proteins used in the present study. $\mathrm{dFv}$-LDP is a fusion protein that comprises LDP and tandem dual Fv segments from the antibody directed against gelatinase. Ec-LDP-Hr is an
EGFR/HER2-bispecific fusion protein in which Ec is an EGFRtargeting oligopeptide and $\mathrm{Hr}$ is a CDR3 sequence from an anti-HER2 antibody. Ec-LDP-Hr-AE, the enediyne-energized fusion protein were prepared by adding AE to Ec-LDP-Hr. We prepared the fusion proteins Ec-LDP-Hr and dFv-LDP as reported $(17,18)$ and analyzed by SDS-PAGE (Fig. 1B and C). The enediyne-energized fusion protein Ec-LDP-Hr-AE was prepared by integrating the enediyne AE into the fusion protein Ec-LDP-Hr as previously described (17).

We first detected the expression levels of EGFR, HER2 and MMP-2 in three pancreatic cancer cell lines (AsPC-1, PANC-1, MIA PaCa-2) and a colorectal cancer cell (HCT-15) by western blotting (Fig. 2A). The results showed that AsPC-1 and PANC-1 cells had higher expression of EGFR while MIA PaCa-2 and HCT-15 cells had higher expression of HER2, respectively. However, the expression levels of MMP-2 in MIA PaCa-2 and HCT-15 were higher than those in AsPC-1 and PANC-1 cells. 
Subsequently, we tested the antiproliferation effects of Ec-LDP-Hr by using MTT assay. The results showed that Ec-LDP-Hr $(50 \mu \mathrm{g} / \mathrm{ml})$ was more potent than LDP $(50 \mu \mathrm{g} / \mathrm{ml})$ on inhibitions of HCT-15 and PANC-1 cell proliferation (Fig. 3A). Due to the antibody-based bispecific fusion protein Ec-LDP-Hr targeting EGFR and HER2, western blotting was used to analyze the change of EGFR/HER2 signaling pathway. As shown in Fig. 3C and D, there was a dose-dependent decrease of the expression and the phosphorylation level of proteins in the EGFR/HER2 signaling pathway in colorectal cancer HCT-15 cells while this was not observed in PANC-1 cells. These results suggested that the efficacy of proliferation inhibition by Ec-LDP-Hr was through EGFR/HER2 signal pathway by HER2 targeting peptide $\mathrm{Hr}$ from CDR3 region.

Moreover, we found that the MMP-2 secreted from fibrosarcoma HT1080 cells in their culture supernatant was 6-fold more than HCT-15 cells analyzed by ELISA (Fig. 2E). After cultivating for $24 \mathrm{~h}$, the culture of HCT-15 in 96-well plates was replaced with HT1080 culture supernatant adding 10\% FBS and exposed to dFv-LDP $(100 \mu \mathrm{g} / \mathrm{ml})$. The result showed that HT1080 culture supernatant promoted HCT-15 cell proliferation. However, this effect was attenuated by adding MMP-2/9 targeting protein $\mathrm{dFv}$-LDP (Fig. 2F). The results suggested that dFv-LDP inhibited the growth of HCT-15 cells by blocking the action of surrounding MMP-2 on the cancer cells.

Binding capability of $d F v-L D P$ and Ec-LDP-Hr to human colorectal cancer HCT-15 cells. The binding capability of $\mathrm{dFv}$-LDP and Ec-LDP-Hr to colorectal cancer cells HCT-15 was examined by ELISA (Fig. 2A) and cell immunofluorescence (Fig. 2B). The results showed that dFv-LDP and Ec-LDP-Hr were able to bind to the HCT-15 cells more markedly compared with LDP. Also, dFv-LDP had stronger binding ability than Ec-LDP-Hr. To further confirm the ability of dFv-LDP and Ec-LDP-Hr binding to MMP-2 and EGFR/HER2 respectively, coimmunoprecipitation assay was carried out. As shown in Fig. 3C and D, the targeting fusion proteins $\mathrm{dFv}$-LDP and Ec-LDP-Hr were precipitated as part of the complex with MMP-2 (lane C3), EGFR (lane D3) and HER2 (lane D4). By laser scanning confocal assay, as shown in the Fig. 2E, Ec-LDP-Hr (red fluorescence) was found to co-localize with HER2 (green fluorescence) and their merge (yellow fluorescence) was localized on the cell membrane.

Combinations of $d F v-L D P$ with Ec-LDP-Hr on HCT-15 xenograft in vivo. The first experiment in vivo was set to determine the synergistic effect of the fusion proteins $\mathrm{dFv}-\mathrm{LDP}$ and Ec-LDP-Hr on colorectal cancer HCT-15 xenograft in nude mice. The fusion proteins $\mathrm{dFv}-\mathrm{LDP}(20 \mathrm{mg} / \mathrm{kg})$ or Ec-LDP-Hr $(20 \mathrm{mg} / \mathrm{kg}$ ) alone, and the combination of various dosages including $5,10,20 \mathrm{mg} / \mathrm{kg}$ respectively, were tested. Cetuximab at $20 \mathrm{mg} / \mathrm{kg}$ was used for comparison. As evaluated by tumor volume, the inhibitory rates of Ec-LDP-Hr or dFv-LDP alone were 23.6 and $43.2 \%$, respectively. The inhibitory rates of the combination of dFv-LDP and Ec-LDP-Hr at dosages of 5, 10, $20 \mathrm{mg} / \mathrm{kg}$ were $40.4,53.8$ and $67.5 \%$, respectively (Fig. 4A). The groups treated with a combination of two fusion proteins showed significant statistical difference from control $(\mathrm{P}<0.01)$. The combination treatment with fusion protein Ec-LDP-Hr $(20 \mathrm{mg} / \mathrm{kg})$ and $\mathrm{dFv}-\mathrm{LDP}(20 \mathrm{mg} / \mathrm{kg})$ revealed a synergistic
A

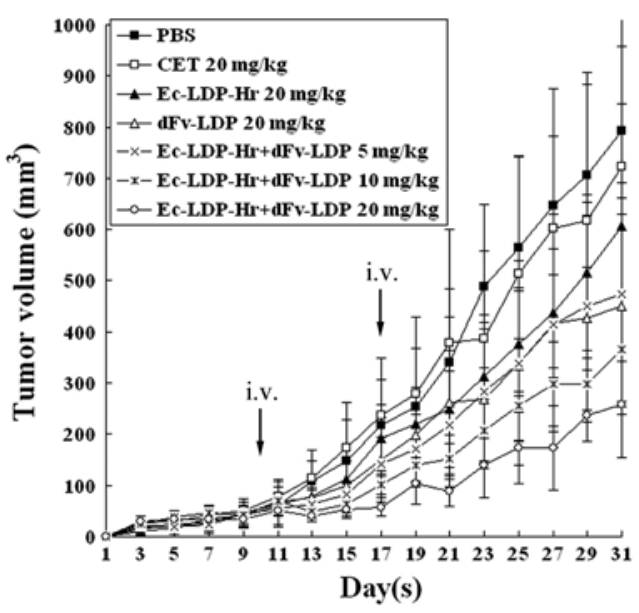

$\mathrm{B}$

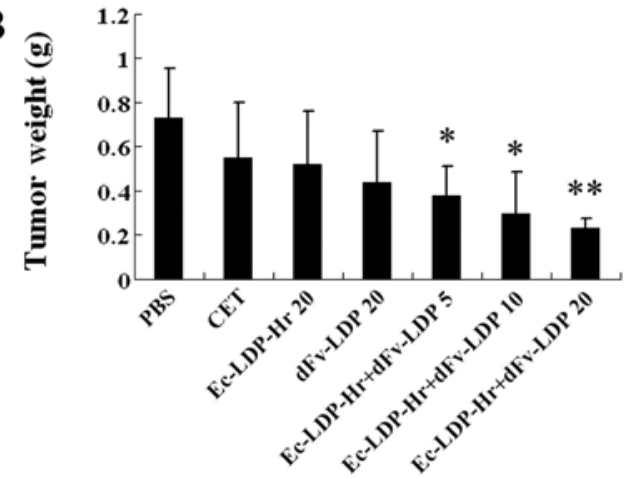

C

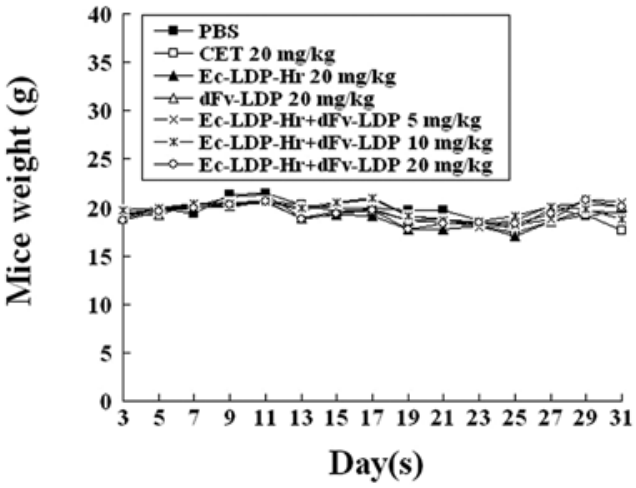

Figure 4. Combinations of dFv-LDP with Ec-LDP-Hr enhance the antitumor efficacy compared with administration alone on HCT-15 xenograft in vivo. The antitumor efficacy of fusion proteins Ec-LDP-Hr and dFv-LDP and the combination in nude mice was tested. The tumor growth (A) curves, (B) tumor weights and (C) body weights of mice are shown. ${ }^{*} \mathrm{P}<0.05$ and ${ }^{* *} \mathrm{P}<0.01$ compared with control.

effect (CDI <0.8). As is known, cetuximab is a human-mouse chimeric $\mathrm{mAb}$ that binds to the extracellular domain of the EGFR (HER1) molecule and is currently approved for clinical use to treat colorectal cancer. In this experiment, cetuximab did not show antitumor efficacy on HCT-15 xenograft in nude mice. At the end of the experiment, the tumors that were excised from the mice were weighed. Similar synergistic tumor inhibition by the combination of dFv-LDP and Ec-LDP-Hr was observed (Fig. 4B). The body weight loss of treated mice was $<10 \%$ of the initial weight, considering that the treatment dosage was tolerated (Fig. 4C). 
A

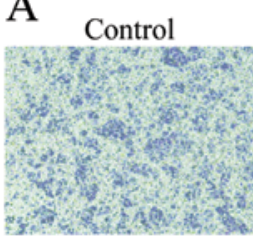

dFv-LDP

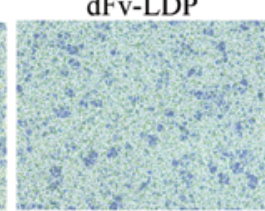

C

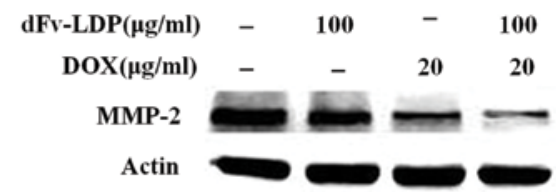

D

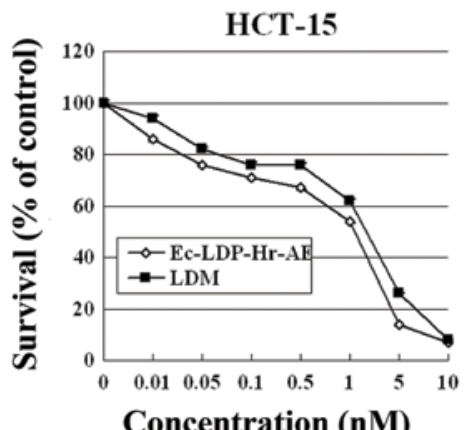

F

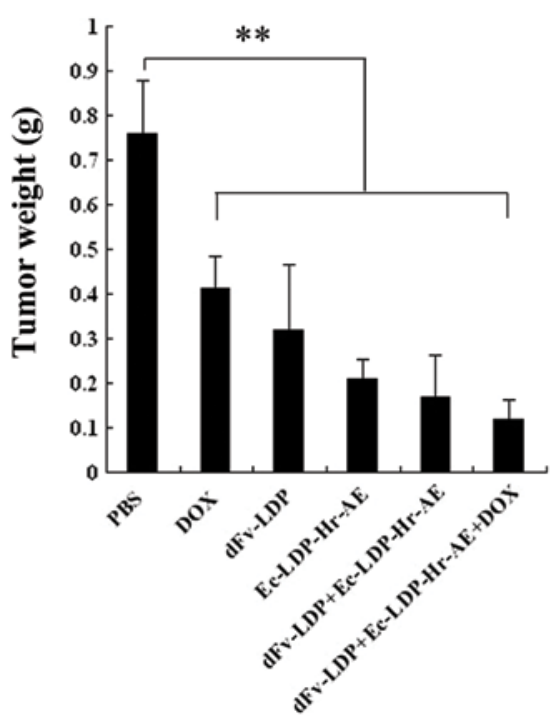

DOX

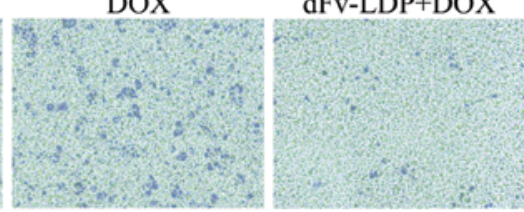

B

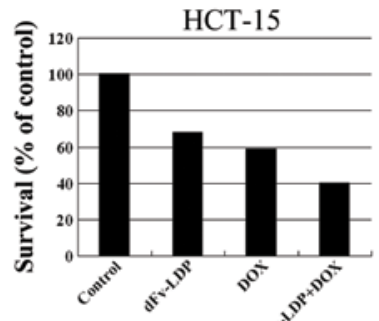

E
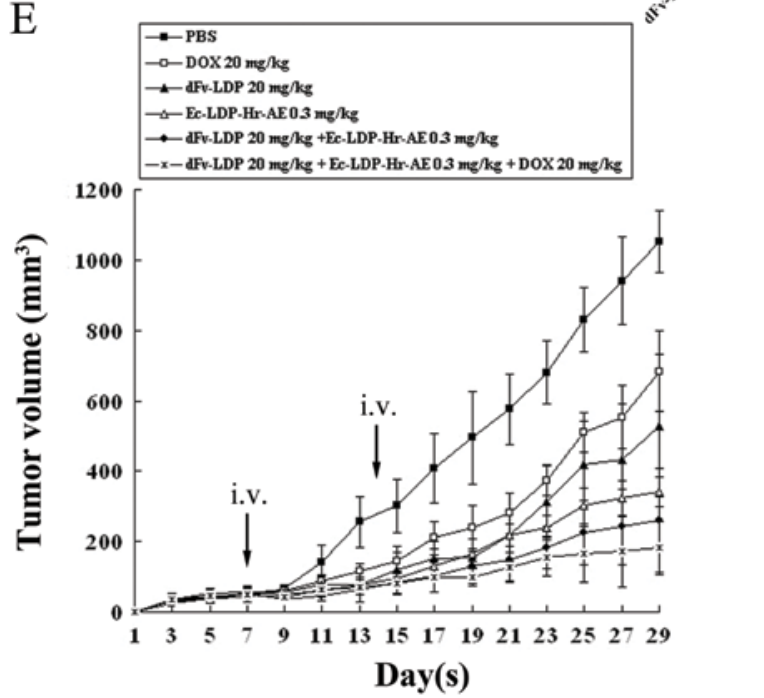

G

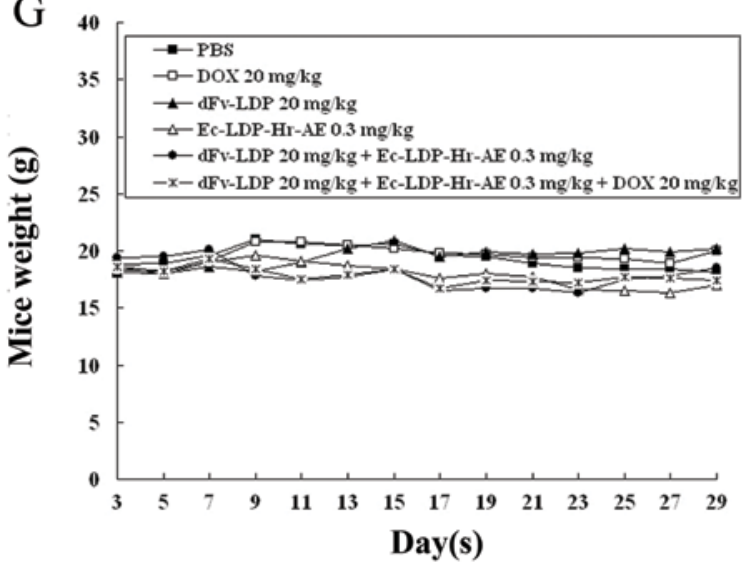

Figure 5. Combinations of dFv-LDP with enediyne-energized fusion protein Ec-LDP-Hr-AE plus doxycycline (DOX) showed more effects in vivo. Synergistically inhibitory effect of dFv-LDP $(100 \mu \mathrm{g} / \mathrm{ml})$ and DOX $(1 \mu \mathrm{g} / \mathrm{ml})$ on HCT-15 cell migration was recorded by (A) camera and was measured by (B) a microplate reader. Enhanced inhibition of MMP-2 expression was detected by dFv-LDP plus DOX in HCT-15 cells as analyzed by western blotting (C). Highly potent cytotoxicity of Ec-LDP-Hr-AE and LDM to HCT-15 cells for $48 \mathrm{~h}$ exposure was determined by (D) MTT assay. Antitumor efficacy of fusion protein $\mathrm{dFv}$-LDP, energized fusion protein Ec-LDP-Hr-AE or DOX alone or in combinations was evaluated by (E) mean tumor volumes and (F) tumor weights; ${ }^{* *} \mathrm{P}<0.01$ compared with control. (G) Body weights of treated mice were recorded.

Combinations of $d F v$-LDP with enediyne-energized fusion protein Ec-LDP-Hr-AE plus DOX on HCT-15 xenograft in vivo. The cytotoxicity of the antibody-based bispecific, enediyne-energized fusion protein Ec-LDP-Hr-AE on HCT-15 cell line was determined by MTT assay. As shown in Fig. 5D, Ec-LDP-Hr-AE displayed highly potent cytotoxicity to cultured HCT-15 cells and was generally more active than the lidamycin. For HCT-15 cells, the $\mathrm{IC}_{50}$ value was $\sim 1 \mathrm{nM}$.
To further improve the antitumor efficacy on colorectal cancer HCT-15 xenograft in nude mice, another experiment was set to enhance the antitumor efficacy by a combination strategy that comprised MMP-2/9-targeting fusion protein $\mathrm{dFv}$-LDP and EGFR/HER2-bispecific enediyne-energized fusion protein Ec-LDP-Hr-AE. For even higher antitumor efficacy, DOX was added to the combination. As shown in Fig. 5E, DOX (20 mg/kg, i.p. administered every other day), 
A

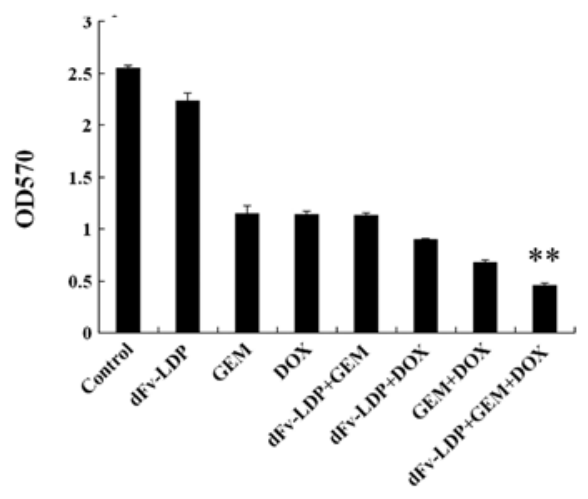

$\mathrm{B}$

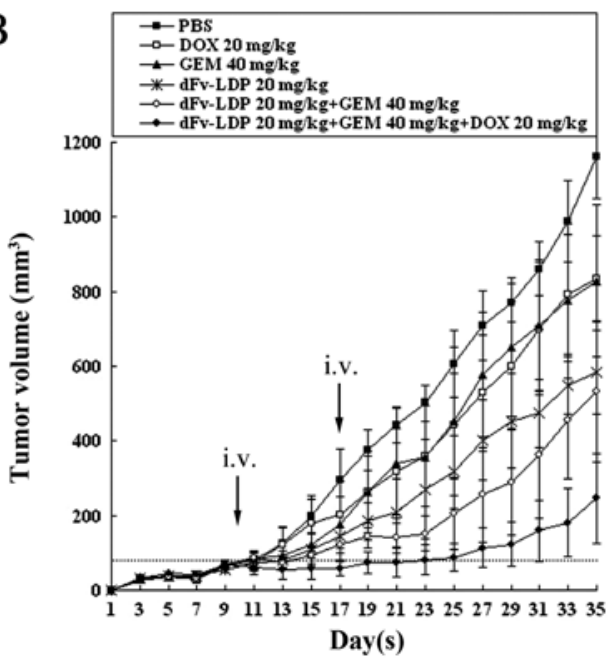

$\mathrm{C}$

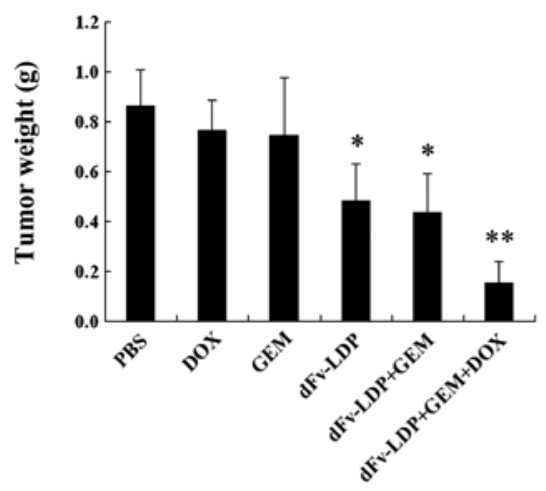

$\mathrm{D}$

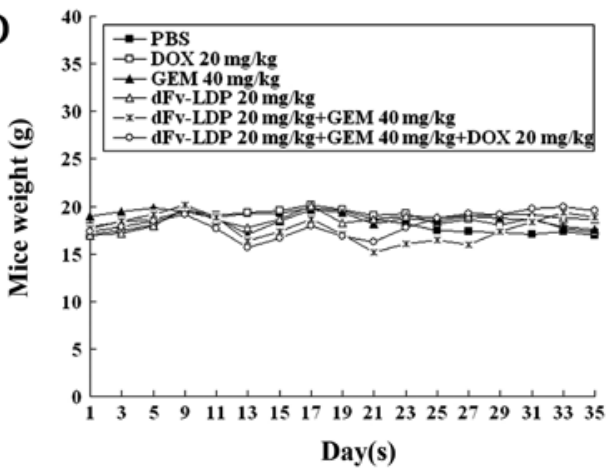

Figure 6. Combinations of $\mathrm{dFv}-\mathrm{LDP}$ with gemcitabine (GEM) and doxycycline (DOX) display enhanced antitumor efficacy against HCT-15 xenograft in vivo. The proliferation inhibition of HCT-15 cells treated with $\mathrm{dFv}-\mathrm{LDP}$ $(200 \mu \mathrm{g} / \mathrm{ml})$, GEM $(0.05 \mu \mathrm{M})$, DOX $(10 \mu \mathrm{g} / \mathrm{ml})$ or the combinations for $72 \mathrm{~h}$ was determined by (A) MTT assay. Antitumor efficacy of fusion protein dFvLDP, GEM or DOX alone or combinations was evaluated by (B) mean tumor volumes and (C) tumor weights. (D) The body weight of treated mice was recorded. ${ }^{*} \mathrm{P}<0.05,{ }^{* * *} \mathrm{P}<0.01$ compared with control.
dFv-LDP (20 mg/kg) or Ec-LDP-Hr-AE (0.3 mg/kg) alone displayed antitumor effects with inhibitory rates of $35,49.7$ and $67.5 \%$, respectively. The inhibitory rate of combination of dFv-LDP and Ec-LDP-Hr-AE was 75.1\%. Furthermore, this combination plus DOX showed stronger efficacy with an inhibitory rate of $82.7 \%$. Similar antitumor efficacy was determined by measurement of tumor weights (Fig. 5F). The groups treated with agents in this experiment showed significant statistical difference from control $(\mathrm{P}<0.01)$. The body weight loss in mice treated with Ec-LDP-Hr-AE was $<10 \%$ of the initial body weight, considering that the treatment dosage was tolerated (Fig. 5G).

Combinations of $d F v-L D P$ with GEM and DOX display enhanced antitumor efficacy against HCT-15 xenograft in vivo. GEM as a chemotherapeutic drug has been used for several types of cancer. Here, the cytotoxicity of GEM, dFv-LDP, DOX, and their combinations to HCT-15 cells were determined by MTT assay (Fig. 6A). The results showed that the combination of $\mathrm{dFv}$-LDP $(200 \mu \mathrm{g} / \mathrm{ml})$ with GEM $(0.05 \mu \mathrm{M})$ and DOX $(10 \mu \mathrm{g} / \mathrm{ml})$ was more potent than any of the two drugs combined or alone respectively, in cytotoxicity to HCT-15 cells.

The antitumor efficacy by a combination strategy that comprises dFv-LDP, GEM and DOX was analyzed on HCT-15 xenograft in vivo. As shown in Fig. 6B, dFv-LDP (20 mg/kg), DOX $(20 \mathrm{mg} / \mathrm{kg})$ or GEM (40 mg/kg) alone displayed antitumor effects with inhibitory rates of $47 ., 27.2$, or $25.3 \%$ at day 25 , respectively. The inhibitory rate of the combination of dFv-LDP and GEM was 66.3\%. Furthermore, this combination plus DOX displayed notable antitumor efficacy, producing initial shrinkage after the first administration and the tumor inhibitory rate was $85.5 \%$ at day 25 , whereas there was no tumor shrinkage observed in any other group. Similar antitumor efficacy was determined by measurement of tumor weights (Fig. 6C). The body weight loss of treated mice was $<10 \%$ of the initial weight, considering that the treatment dosage was tolerated (Fig. 6D).

\section{Discussion}

At present, the vast majority of biomarker research has focused on targeted therapies. Efforts are continuing to identify predictive markers of response or resistance to chemotherapy (23). One of the most promising targets for antitumor therapy is the epithelial growth factor receptor (EGFR) family in the treatment of metastatic cancer $(24,25)$. Several studies have shown that agents targeting the EGFR improve outcomes in cancer $(26,27)$. Currently, two anti-EGFR monoclonal antibodies, panitumumab (an IgG2 type human antibody directed against the same epitope) and cetuximab (an IgG3 type chimeric antibody directed against the extra-cellular portion of the receptor), have been clinically validated for the treatment of metastatic colorectal cancer.

In addition, matrix metalloproteinases (MMPs) play an important role in cancer progression and spread such as invading surrounding tissues, basement membranes and extracellular matrix (28). In colorectal cancer, MMP-2 immunoexpression associates with advanced disease $(29,30)$, and high MMP-2 expression in cancer cells and the stroma associates with poor prognosis (31). MMP-9 correlates with 
metastatic disease and poor prognosis (32). For the abundance of MMP-2/9 increases in colorectal carcinoma, highly selective MMP inhibitors should also be potential therapeutics (33). As is well known, MMP-2/9 is generally overexpressed in highly invasive and metastatic cancer cells, in cancer associated fibroblast and in proliferating endothelial cells. Therefore, MMP-2/9 appears to be a highly attractive, multi-faceted target in cancer therapy. Targeting MMP-2/9 may actually impose actions on both cancer cells and stromal cells, leading to cancer cell killing and modulation of the tumor microenvironment. It is of particular interest to develop a new strategy for drug combinations on the basis of targeting MMP-2/9.

Globally, colorectal cancer is the third most commonly diagnosed cancer in males and the second in females (34). Approximately one-quarter of CRC patients have metastases at diagnosis and a further 33-50\% develop metastases over their disease course (35). Chemotherapy has been the standard care for metastatic colorectal cancer patients for many years and is based mainly on the use of three agents: 5-fluorouracil, irinotecan and oxaliplatin. The role of other chemotherapeutic drugs such as gemcitabine (GEM) has not yet been established (18). Evidence has shown that GEM was more potent and had considerably broader antitumor activity against various murine tumors (myeloma, adenocarcinoma, ovarian carcinoma, lymphosarcoma and leukemia) and human tumor xenograft models (breast, colon, lung and pancreatic) (36). In the present study, we set up the colorectal cancer HCT-15 xenograft in athymic mice to evaluate the efficacy of various combinations.

In previous studies, we reported two specific targeted fusion proteins, dFv-LDP and Ec-LDP-Hr $(16,17)$, which had strong affinity and activity to cancer cells overexpressing MMP-2/9 and EGFR/HER2, respectively. dFv-LDP is an MMP-2/9-specific dFv-based fusion protein. Ec-LDP-Hr is an EGFR/HER2-bispecific fusion protein and energized Ec-LDP-Hr-AE belongs to its corresponding antibody-drug conjugates (ADCs) in which the extremely potent cytotoxic enediyne AE serves as 'warhead' agent. Here, we demonstrated that the targeting proteins dFv-LDP and Ec-LDP-Hr had strong binding abilities to colorectal cancer cells. Both of them had antiproliferation effects of cancer cells in vitro and therapeutic efficacy in vivo. The present investigations evaluated several settings of combinations including i) dFv-LDP plus the EGFR/HER2-bispecific fusion protein Ec-LDP-Hr, ii) dFv-LDP plus the EGFR/HER2-bispecific and enediyneenergized fusion protein Ec-LDP-Hr-AE, iii) dFv-LDP plus Ec-LDP-Hr-AE and DOX, and iv) dFv-LDP plus GEM and DOX. The results indicated that $\mathrm{dFv}$-LDP enhanced antitumor efficacy against colorectal cancer xenograft when combined with EGFR/HER2-targeted fusion protein or the chemotherapeutic GEM. Further enhanced efficacy can be achieved by the addition of DOX.

In summary, the MMP-2/9-targeting fusion protein $\mathrm{dFV}$-LDP plus DOX enhances antitumor efficacy in combination respectively, with EGFR/HER2-bispecific fusion protein, its corresponding enediyne-energized fusion protein, and the chemotherapeutic GEM. This MMP-2/9-oriented combination strategy that uses MMP-2/9-targeting antibody-based fusion protein and the small molecular inhibitor DOX as the basic composed agents may be effective in cancer therapy.

\section{Acknowledgements}

The authors acknowledge the 'Significant New Drugs Development' Major Science and Technology Projects of China (nos. 2013ZX09102064 and 2012ZX09301002-001-015).

\section{References}

1. Sapra P and Shor B: Monoclonal antibody-based therapies in cancer: advances and challenges. Pharmacol Ther 138: 452-469, 2013.

2. Pedersen MW, Jacobsen HJ, Koefoed K, Hey A, Pyke C, Haurum JS and Kragh M: Sym004: a novel synergistic antiepidermal growth factor receptor antibody mixture with superior anticancer efficacy. Cancer Res 70: 588-597, 2010.

3. Sapra P, Damelin M, Dijoseph J, et al: Long-term tumor regression induced by an antibody-drug conjugate that targets 5T4, an oncofetal antigen expressed on tumor-initiating cells. Mol Cancer Ther 12: 38-47, 2013.

4. Maron R1, Schechter B, Mancini M, Mahlknecht G, Yarden Y and Sela M: Inhibition of pancreatic carcinoma by homo- and heterocombinations of antibodies against EGF-receptor and its kin HER2/ErbB-2. Proc Natl Acad Sci USA 110: 15389-15394, 2013.

5. McCaffery I, Tudor Y, Deng H, et al: Putative predictive biomarkers of survival in patients with metastatic pancreatic adenocarcinoma treated with gemcitabine and ganitumab, an IGF1R inhibitor. Clin Cancer Res 19: 4282-4289, 2013.

6. Shankaran V, Obel J and Benson AD III: Predicting response to EGFR inhibitors in metastatic colorectal cancer: current practice and future directions. Oncologist 15: 157-167, 2010.

7. Normanno N, De Luca A, Bianco C, et al: Epidermal growth factor receptor (EGFR) signaling in cancer. Gene 366: 2-16, 2006.

8. Hynes NE and Lane HA: ERBB receptors and cancer: the complexicity of targeted inhibitors. Nat Rev Cancer 5: 341-354, 2005.

9. Harrison $\mathrm{S}$ and Benziger $\mathrm{H}$ : The molecular biology of colorectal carcinoma and its implications: a review. Surgeon 9: 200-210, 2011.

10. Saxby AJ, Nielsen A, Scarlett CJ, Clarkson A, Morey A, Gill A and Smith RC: Assessment of HER-2 status in pancreatic adenocarcinoma: correlation of immunohistochemistry, quantitative real-time RT-PCR, and FISH with aneuploidy and survival. Am J Surg Pathol 29: 1125-1134, 2005.

11. Yamanaka Y, Friess H, Kobrin MS, Buchler M, Beger HG and Korc M: Coexpression of epidermal growth factor receptor and ligands in human pancreatic cancer is associated with enhanced tumor aggressiveness. Anticancer Res 13: 565-569, 1993.

12. Björklund $M$ and Koivunen E: Gelatinase-mediated migration and invasion of cancer cells. Biochim Biophys Acta 1755: 37-69, 2005.

13. Kallakury BV, Karikehalli S, Haholu A, Sheehan CE, Azumi N and Ross JS: Increased expression of matrix metalloproteinases 2 and 9 and tissue inhibitors of metalloproteinases 1 and 2 correlate with poor prognostic variables in renal cell carcinoma. Clin Cancer Res 7: 3113-3119, 2001.

14. Cho YB, Lee WY, Song SY, Shin HJ, Yun SH and Chun HK: Matrix metalloproteinase-9 activity is associated with poor prognosis in T3-T4 node-negative colorectal cancer. Hum Pathol 38: 1603-1610, 2007.

15. Hong SW, Kang YK, Lee B, et al: Matrix metalloproteinase-2 and -7 expression in colorectal cancer. J Korean Soc Coloproctol 27: 133-139, 2011.

16. Guo XF, Zhu XF, Shang Y, Zhang SH and Zhen YS: A bispecific enediyne-energized fusion protein containing ligand-based and antibody-based oligopeptides against epidermal growth factor receptor and human epidermal growth factor receptor 2 shows potent antitumor activity. Clin Cancer Res 16: 2085-2094, 2010.

17. Zhong G, Zhang S, Li Y, Liu X, Gao R, Miao Q and Zhen Y: A tandem scFv-based fusion protein and its enediyne-energized analogue show intensified therapeutic efficacy against lung carcinoma xenograft in athymic mice. Cancer Lett 295: 124-133, 2010.

18. Saif MW, Kaley K, Penney R, Hotchkiss S, Syrigos KN and Strimpakos AS: The efficacy of gemcitabine as salvage treatment in patients with refractory advanced colorectal cancer (CRC): a single institution experience. Anticancer Res 31: 2971-2974, 2011. 
19. Pelosof L, Yerram SR, Ahujia N, Delmas A, Danilova L, Herman JG and Azad NS: CHFR silencing or microsatellite instability is associated with increased antitumor activity of docetaxel or gemcitabine in colorectal cancer. Int J Cancer 134: 596-605, 2014.

20. Shen LC, Chen YK, Lin LM and Shaw SY: Anti-invasion and anti-tumor growth effect of doxycycline treatment for human oral squamous-cell carcinoma - in vitro and in vivo studies. Oral Oncol 46: 178-184, 2010.

21. Guimaraes DA, Rizzi E, Ceron CS, et al: Doxycycline dosedependently inhibits MMP-2-mediated vascular changes in 2K1C hypertension. Basic Clin Pharmacol Toxicol 108: 318-325, 2011.

22. Son K, Fujioka S, Iida T, et al: Doxycycline induces apoptosis in PANC-1 pancreatic cancer cells. Anticancer Res 29: 3995-4004, 2009.

23. Heinemann V, Douillard JY, Ducreux M and Peeters M: Targeted therapy in metastatic colorectal cancer - an example of personalized medicine in action. Cancer Treat Rev 39: 592-601, 2013.

24. Grünwald V and Hidalgo M: Developing inhibitors of the epidermal growth factor receptor for cancer treatment. J Nat Cancer Inst 95: 851-867, 2003.

25. Jones S, Zhang X, Parsons DW, et al: Core signaling pathways in human pancreatic cancers revealed by global genomic analyses. Science 321: 1801-1806, 2008.

26. Winder T and Lenz HJ: Vascular endothelial growth factor and epidermal growth factor signaling pathways as therapeutic targets for colorectal cancer. Gastroenterology 138: 2163-2176, 2010.

27. Medinger M and Drevs J: Receptor tyrosine kinases and anticancer therapy. Curr Pharm Des 11: 1139-1149, 2005.
28. Coussens LM and Werb Z: Matrix metalloproteinases and the development of cancer. Chem Biol 3: 895-904, 1996.

29. Matsuyama Y, Takao S and Aikou T: Comparison of matrix metalloproteinase expression between primary tumors with or without liver metastasis in pancreatic and colorectal carcinomas. J Surg Oncol 80: 105-110, 2002.

30. Papadopoulou S, Scorilas A, Arnogianaki N, Papapanayiotou B, Tzimogiani A, Agnantis $\mathrm{N}$ and Talieri M: Expression of gelatinase-A (MMP-2) in human colon cancer and normal colon mucosa. Tumour Biol 22: 383-389, 2001.

31. Hilska M, Roberts PJ, Collan YU, et al: Prognostic significance of matrix metalloproteinases-1, $-2,-7$ and -13 and tissue inhibitors of metalloproteinases-1, $-2,-3$ and -4 in colorectal cancer. Int J Cancer 121: 714-723, 2007.

32. Karakiulakis G, Papanikolaou C, Jankovic SM, Aletras A, Papakonstantinou E, Vretou E and Mirtsou-Fidani V: Increased type IV collagen-degrading activity in metastases originating from primary tumors of the human colon. Invasion Metastasis 17: 158-168, 1997.

33. Roeb E, Dietrich CG, Winograd R, et al: Activity and cellular origin of gelatinases in patients with colon and rectal carcinoma differential activity of matrix metalloproteinase-9. Cancer 92: 2680-2691, 2001

34. Jemal A, Bray F, Center MM, Ferlay J, Ward E and Forman D: Global cancer statistics. CA Cancer J Clin 61: 69-90, 2011.

35. Garden OJ, Rees M, Poston GJ, et al: Guidelines for resection of colorectal cancer liver metastases. Gut 55 (Suppl 3): iiil-iii8, 2006.

36. Gesto DS1, Cerqueira NM, Fernandes PA and Ramos MJ: Gemcitabine: a critical nucleoside for cancer therapy. Curr Med Chem 19: 1076-1087, 2012. 